\title{
Response of Soybean Accessions from Provinces in Southern China to Phytophthora sojae
}

\author{
D. E. Kyle, Graduate Research Fellow, C. D. Nickell, Professor of Plant Breeding, and R. L. Nelson, Supervisory \\ Research Geneticist and Associate Professor of Plant Genetics, USDA-Agricultural Research Service, Plant Physi- \\ ology and Genetics Research Unit; and W. L. Pedersen, Associate Professor of Crop Sciences, Department of Crop \\ Sciences, University of Illinois, Urbana 61801
}

\begin{abstract}
Kyle, D. E., Nickell, C. D., Nelson, R. L., and Pedersen, W. L. 1998. Response of soybean accessions from provinces in southern China to Phytophthora sojae. Plant Dis. 82:555-559.

Phytophthora rot, caused by Phytophthora sojae, is a damaging disease of soybean (Glycine $\max ($ L.) Merr.) throughout the soybean-producing regions of the world. The discovery of new sources of resistance in soybean is vital in maintaining control of Phytophthora rot, because races of the pathogen have been discovered that can attack cultivars with commonly used resistance genes. The objectives of this study were to investigate the distribution and diversity of Phytophthora-resistant soybean in southern China and identify sources that confer resistance to multiple races for implementation into breeding programs. Soybean accessions obtained from southern China were evaluated for their response to races $1,3,4,5,7,10,12,17,20$, and 25 of P. sojae using the hypocotyl inoculation technique in the greenhouse at Urbana, Illinois in 1996 and 1997. Accessions were identified that confer resistant responses to multiple races of the pathogen. These accessions may provide sources of resistance for control of Phytophthora rot of soybean in the future. The majority of the accessions with resistance to eight or more of the ten races tested were from the provinces of Hubei, Jiangsu, and Sichuan in southern China. Based on the evaluated accessions, these provinces appear to be valuable sources of Phytophthoraresistant soybean.
\end{abstract}

Phytophthora rot of soybean, caused by Phytophthora sojae (14), is a problem in the major soybean producing countries of the world $(28,34)$ and has been reported as the second most damaging disease to soybean in the north central United States (12). The disease was first reported in association with soybean in 1955 by Kaufmann and Gerdemann (16). Since that time, 45 physiologic races of $P$. sojae have been reported $(1,15)$. Thirteen single dominant genes and alleles condition resistance to specific races of $P$. sojae (30). These genes have provided adequate disease control in the past, but extensive usage has promoted selection of pathogen races capable of overcoming these resistance genes. Future control may not be possible unless new sources of resistance are identified $(26,30,32)$.

Lohnes et al. (18) evaluated 726 accessions from central China (plant introduc-

Corresponding author: C. D. Nickell

E-mail: cnickell@uiuc.edu

Contribution of the Illinois Agricultural Experimental Station Research supported in part by the Illinois Soybean Program Operating Board and the United Soybean Board.

Accepted for publication 2 February 1998.

Publication no. D-1998-0309-02R

(c) 1998 The American Phytopathological Society tion numbers [PI] 567.290 to 567.781) for reaction to races $1,3,7$, and 25 of $P$. sojae. They reported that a high frequency of Phytophthora-resistant accessions occurred in central China, especially from the provinces of Anhui and Jiangsu. The reaction patterns of the resistant accessions were found to be extremely diverse. Phytophthora rot of soybean was not reported in China until 1993 (35). However, Lohnes et al. (18) attributed the distribution and diversity of Phytophthora resistance in soybean accessions to the evolution of Phytophthora spp. and soybean.

The objectives of this study were to investigate the distribution of Phytophthoraresistant accessions from provinces in southern China, assess the diversity of reaction patterns from resistant accessions to multiple races of $P$. sojae, and distinguish accessions with resistance to multiple races as potential sources of resistance for control of Phytophthora rot in the future.

\section{MATERIALS AND METHODS}

The accessions evaluated from southern China included PI 587.550A to PI 588.053B. Seeds were obtained from the United States Department of Agriculture (USDA) Soybean Germplasm Collection in Urbana, Illinois. Fifty-eight of the total 686 accessions in this range were not included in this report due to a low quantity of available seed or incomplete data on the response to all races. The accessions originated from the provinces of Anhui, Guangdong, Hebei, Jiangsu, Shanghai, Sichuan, and Zhejiang in southern China. Accessions were evaluated for their response to races $1,3,4,5,7,10$, and 20 of $P$. sojae by the hypocotyl inoculation technique using zoospores (20). Accessions were evaluated for their response to races 12,17 , and 25 by the hypocotyl inoculation technique using mycelium (29) at Urbana, Illinois in 1997. Races 12, 17, and 25 did not produce 50,000 zoospores $/ \mathrm{ml}$; therefore, mycelium was used to inoculate plants.

Races $1,3,7,12$, and 20 of $P$. sojae were previously available at the University of Illinois. Races 4, 5, 17, and 25 were obtained from A. F. Schmitthenner (Department of Plant Pathology, Ohio State University). These races were used because they were available and could be used to separate plant responses to compare with known resistant alleles. All races were tested for virulence against a differential set of cultivars. Some differences in virulence of races from the originally reported virulence formulas were found. These differences were attributed to the instability of virulence in mass cultures (30), the variability within pathogen populations and zoospore propagation (27), and the differences in inoculation technique used for classification, so the original race number designations were not changed. The reactions of the differential cultivars to these cultures of each race and the original reported reactions (32) are listed in Table 1.

Modifications were made to the technique of Moots et al. (21) for producing inoculum. The modifications were (i) 5 small pieces of agar with mycelium were transferred to each petri plate (100 by 15 $\mathrm{mm}$ ) containing $8 \mathrm{ml}$ lima bean agar (LBA; Difco dehydrated LBA 23g/liter) and incubated in the dark for 4 to 5 days, and (ii) during the rinsing procedure and the time following the addition of the fifth portion of rinse water, the plates were incubated in the dark at $20^{\circ} \mathrm{C}$. The concentration of zoospores used for inoculation ranged from 74,500 to 107,800 zoospores $/ \mathrm{ml}$. The modifications to the Schmitthenner and Bhat (29) inoculation technique were (i) 5 small pieces of agar with mycelium were transferred to each 
petri plate $(100 \times 15 \mathrm{~mm})$ containing $8 \mathrm{ml}$ LBA and incubated for 4 to 5 days, (ii) agar covered with mycelium was placed in a $60-\mathrm{ml}$ syringe and forced through a $20-$ gauge needle into a 5-ml syringe, and (iii) a 5-ml syringe with a 25 -gauge needle was used to place approximately $75 \mu \mathrm{l}$ inoculum into a slit in the hypocotyl.

Ten seeds of each accession were planted in a $10-\mathrm{cm}$-diameter plastic pot filled with sand. Plants were grown in the greenhouse under a 14-h photoperiod with temperatures ranging from 22 to $30^{\circ} \mathrm{C}$. For every 35 accessions screened, a resistant and susceptible check was included to verify the success of the inoculation. The cultivars Macon (24) and Williams (7) were planted as susceptible standards for all tested races. Other standards were, "Piatt" (23), Williams isolines (L75-3735, L77-1794, L85-2352, and L85-3059; 31), "Chapman" (19), "Harosoy" (33) and Harosoy isolines (Harosoy 12XX, Harosoy 62XX; 11), L70-6494, UCO 112, and Kenwood 94. The response of these standards was dependent upon the resistance gene present in the cultivar and the race used for inoculating. Five days after inoculation, accessions were rated as resistant, susceptible, or intermediate. Response classifications to each race were based on all plants within a single pot for each accession. Resistant classifications were given if greater than $70 \%$ of the plants were alive. Accessions were rated as sus- ceptible if greater than $70 \%$ of the plants were dead. Intermediate classifications were given for accessions that ranged from 70 to $30 \%$ survival.

\section{RESULTS AND DISCUSSION}

Resistance to races of $P$. sojae was relatively common in soybean accessions from southern China. The total number of accessions from each province and the percent of accessions with resistant and intermediate reactions (combined) to races 1, 3, 4, 5, $7,10,12,17,20$, and 25 are listed in Table 2. The highest percentage of accessions with resistant and intermediate reactions occurred in response to race 1 , followed in order by race $5,17,3,10,7,4,25,12$, and 20 . Only $13 \%$ of the total accessions were resistant to race 20 , while $62 \%$ had resistance to race 1 . The provinces of Anhui and Hubei provided the highest percentages of accessions with resistance to race 1 , race 3 , race 7 , race 10 , and race 17 , individually. The provinces of Hubei and Jiangsu provided the highest percentages of accessions with resistance to race 4 . The highest percentage of resistant accessions to race 5 were from the provinces of Hubei, Jiangsu, and Zhejiang. Approximately one-fourth of the accessions from the provinces of Jiangsu and Sichuan had resistance to race 12 . The percentage of accessions with resistance to race 20 was comparably low from all provinces, while the provinces of Jiangsu, Anhui, and
Shanghai had the highest percentages. The percentage of accessions with resistance to race 25 was highest from the provinces of Jiangsu and Zhejiang, and relativity high from Hubei, Shanghai, and Sichuan. The provinces of Anhui, Hubei, Jiangsu, Shanghai, and Zhejiang, which contributed high percentages of resistant accessions to specific races, are located in eastern China. The province of Sichuan, which provided a high percentage of accessions with resistance to race 12 , is located in the central region of China. Accessions from northern Anhui and Jiangsu were also included in the evaluation by Lohnes et al. (18). In both that study and this research, accessions from these two provinces had high percentages of resistant accessions to specific races, further exemplifying their value as sources for Phytophthora-resistant accessions. It is probable that the greatest Phytophthora pressure is occurring in these areas of China; however, this has not been verified with isolation of $P$. sojae from China. The high frequency of resistant accessions to a specific race can likely be attributed to the presence of that race or races with similar virulence in that region of China. This information also provides direction for collecting soybean with resistance to specific races of $P$. sojae.

Accessions from southern China elicited 147 different reaction types, out of the total 1,024 reaction types that are possible with 10 race reactions. Reaction types are based

Table 1. Response of differential cultivars to cultures of Phytophthora sojae used for testing

\begin{tabular}{|c|c|c|c|c|c|c|c|c|c|c|c|}
\hline \multirow[b]{2}{*}{ Cultivar } & \multirow[b]{2}{*}{ Gene(s) } & \multicolumn{10}{|c|}{ Race of $P$ soja $e^{\mathrm{a}}$} \\
\hline & & 1 & 3 & 4 & 5 & 7 & 10 & 12 & 17 & 20 & 25 \\
\hline Williams & $r p s$ & $\mathrm{~S}(\mathrm{~S})^{\mathrm{b}}$ & $\mathrm{S}(\mathrm{S})$ & $\mathrm{S}(\mathrm{S})$ & $\mathrm{S}(\mathrm{S})$ & $\mathrm{S}(\mathrm{S})$ & $\mathrm{S}(\mathrm{S})$ & $S(S)$ & $\mathrm{S}(\mathrm{S})$ & $\mathrm{S}(\mathrm{S})$ & $\mathrm{S}(\mathrm{S})$ \\
\hline Mukden & Rps1 & $\mathrm{R}(\mathrm{R})$ & $S(S)$ & $S(S)$ & $S(S)$ & $S(S)$ & $\mathrm{R}(\mathrm{R})$ & $S(S)$ & $\mathrm{R}(\mathrm{R})$ & $S(S)$ & $S(S)$ \\
\hline Sanga & Rps $1 b$ & $\mathrm{R}(\mathrm{R})$ & $\mathrm{R}(\mathrm{R})$ & $\mathrm{R}(\mathrm{R})$ & $\mathrm{R}(\mathrm{R})$ & $\mathrm{R}(\mathrm{R})$ & $\mathrm{S}(\mathrm{S})$ & $\mathrm{S}(\mathrm{S})$ & $\mathrm{S}(\mathrm{S})$ & $\mathrm{S}(\mathrm{S})$ & $S(S)$ \\
\hline Arksoy & Rpslc & $\mathrm{R}(\mathrm{R})$ & $\mathrm{R}(\mathrm{R})$ & $\mathrm{S}(\mathrm{S})$ & $S(S)$ & $\mathrm{R}(\mathrm{R})$ & $\mathrm{R}(\mathrm{R})$ & $\mathrm{I}(\mathrm{S})$ & $\mathrm{R}(\mathrm{R})$ & $S(S)$ & $S(S)$ \\
\hline PI 103091 & Rpsld & $\mathrm{R}(\mathrm{R})$ & $\mathrm{R}(\mathrm{R})$ & $\mathrm{R}(\mathrm{R})$ & $\mathrm{R}(\mathrm{R})$ & $\mathrm{R}(\mathrm{R})$ & $\mathrm{R}(\mathrm{R})$ & $\mathrm{R}(\mathrm{S})$ & $\mathrm{S}(\mathrm{S})$ & $\mathrm{R}(\mathrm{R})$ & $\mathrm{R}(\mathrm{R})$ \\
\hline Kingwa & Rpslk & $\mathrm{R}(\mathrm{R})$ & $\mathrm{R}(\mathrm{R})$ & $\mathrm{R}(\mathrm{R})$ & $\mathrm{R}(\mathrm{R})$ & $\mathrm{R}(\mathrm{R})$ & $\mathrm{R}(\mathrm{R})$ & $S(S)$ & $\mathrm{R}(\mathrm{R})$ & $S(S)$ & $\mathrm{S}(\mathrm{S})$ \\
\hline CNS & Rps 2 & $\mathrm{I}(\mathrm{R})$ & $\mathrm{S}(\mathrm{R})$ & $\mathrm{R}(\mathrm{R})$ & $\mathrm{S}(\mathrm{S})$ & $\mathrm{S}(\mathrm{S})$ & $\mathrm{R}(\mathrm{R})$ & $\mathrm{S}(\mathrm{R})$ & $\mathrm{S}(\mathrm{R})$ & $\mathrm{R}(\mathrm{R})$ & $\mathrm{R}(\mathrm{R})$ \\
\hline PI 171442 & Rps $3 a$ & $\mathrm{R}(\mathrm{R})$ & $\mathrm{R}(\mathrm{R})$ & $\mathrm{R}(\mathrm{R})$ & $\mathrm{R}(\mathrm{R})$ & $S(S)$ & S (S) & $S(S)$ & $S(S)$ & $S(S)$ & $R(R)$ \\
\hline PI 172901 & Rps $3 b$ & $\mathrm{R}(\mathrm{R})$ & $\mathrm{R}(\mathrm{R})$ & $R(R)$ & $\mathrm{R}(\mathrm{R})$ & $\mathrm{R}(\mathrm{R})$ & $\mathrm{S}(\mathrm{R})$ & $\mathrm{R}(\mathrm{R})$ & $S(S)$ & $\mathrm{S}(\mathrm{R})$ & $R(R)$ \\
\hline PI 340046 & Rps1k, Rps3c & $\mathrm{R}(\mathrm{R})$ & $\mathrm{R}(\mathrm{R})$ & $\mathrm{R}(\mathrm{R})$ & $\mathrm{R}(\mathrm{R})$ & $\mathrm{R}(\mathrm{R})$ & $\mathrm{R}(\mathrm{R})$ & $\mathrm{R}(\mathrm{R})$ & $\mathrm{R}(\mathrm{R})$ & $\mathrm{S}(\mathrm{R})$ & $\mathrm{R}(\mathrm{R})$ \\
\hline L83-2352 & Rps 4 & $\mathrm{R}(\mathrm{R})$ & $\mathrm{R}(\mathrm{R})$ & $\mathrm{R}(\mathrm{R})$ & $S(S)$ & $S(S)$ & $\mathrm{R}(\mathrm{R})$ & $\mathrm{R}(\mathrm{R})$ & $S(S)$ & $\mathrm{R}(\mathrm{R})$ & $R(R)$ \\
\hline PI 86050 & Rps1c, Rps4 & $\mathrm{R}(\mathrm{R})$ & $\mathrm{R}(\mathrm{R})$ & $\mathrm{R}(\mathrm{R})$ & $\mathrm{S}(\mathrm{R})$ & $\mathrm{R}(\mathrm{R})$ & $\mathrm{R}(\mathrm{R})$ & $\mathrm{R}(\mathrm{R})$ & $\mathrm{R}(\mathrm{R})$ & $\mathrm{R}(\mathrm{R})$ & $\mathrm{R}(\mathrm{R})$ \\
\hline PI 91160 & Rps 5 & $\mathrm{R}(\mathrm{R})$ & $\mathrm{R}(\mathrm{R})$ & $\mathrm{S}(\mathrm{R})$ & $\mathrm{R}(\mathrm{R})$ & $S(S)$ & $S(S)$ & $S(R)$ & $S(S)$ & $\mathrm{S}(\mathrm{R})$ & $R(R)$ \\
\hline Altona & Rps6 & $\mathrm{R}(\mathrm{R})$ & $\mathrm{R}(\mathrm{R})$ & $\mathrm{R}(\mathrm{R})$ & $\mathrm{S}(\mathrm{S})$ & $\mathrm{S}(\mathrm{S})$ & $\mathrm{R}(\mathrm{R})$ & $\mathrm{R}(\mathrm{R})$ & $\mathrm{S}(\mathrm{S})$ & $\mathrm{R}(\mathrm{R})$ & $R(R)$ \\
\hline Harosoy & Rps 7 & $\mathrm{~S}(\mathrm{~S})$ & $\mathrm{S}(\mathrm{S})$ & $\mathrm{S}(\mathrm{S})$ & $S(S)$ & $\mathrm{S}(\mathrm{S})$ & $\mathrm{S}(\mathrm{S})$ & $\mathrm{I}(\mathrm{R})$ & $\mathrm{S}(\mathrm{S})$ & $\mathrm{S}(\mathrm{S})$ & $S(S)$ \\
\hline
\end{tabular}

${ }^{\mathrm{a}} \mathrm{R}=$ resistant, $\mathrm{S}=$ susceptible, and $\mathrm{I}=$ intermediate.

b Original classification in parentheses (31).

Table 2. Percent of accessions from each province with resistant or intermediate reactions to specific races of Phytophthora sojae

\begin{tabular}{|c|c|c|c|c|c|c|c|c|c|c|c|}
\hline \multirow[b]{2}{*}{ Province } & \multirow[b]{2}{*}{ Total } & \multicolumn{10}{|c|}{ Resistant and intermediate reactions (\%) } \\
\hline & & Race 1 & Race 3 & Race 4 & Race 5 & Race 7 & Race 10 & Race 12 & Race 17 & Race 20 & Race 25 \\
\hline Anhui & 69 & 72 & 59 & 33 & 43 & 49 & 61 & 16 & 61 & 14 & 22 \\
\hline Guangdong & 9 & 11 & 11 & 11 & 11 & 0 & 11 & 11 & 11 & 11 & 11 \\
\hline Hubei & 173 & 80 & 62 & 44 & 68 & 50 & 52 & 18 & 57 & 9 & 28 \\
\hline Jiangsu & 167 & 65 & 46 & 40 & 59 & 34 & 42 & 26 & 44 & 19 & 32 \\
\hline Shanghai & 7 & 29 & 14 & 29 & 29 & 14 & 14 & 14 & 0 & 14 & 29 \\
\hline Sichuan & 130 & 44 & 32 & 29 & 40 & 34 & 25 & 25 & 50 & 12 & 30 \\
\hline Zhejiang & 73 & 48 & 40 & 26 & 52 & 33 & 16 & 16 & 37 & 10 & 32 \\
\hline Total & 628 & 62 & 47 & 36 & 54 & 39 & 40 & 21 & 49 & 13 & 29 \\
\hline
\end{tabular}


Table 3. Distribution of reaction to races 1, 3, 4, 5, 7, 10, 12, 17, 20, and 25 of Phytophthora sojae in soybean accessions from southern China

\begin{tabular}{|c|c|c|c|c|c|c|c|c|c|c|c|c|c|c|c|c|}
\hline \multicolumn{10}{|c|}{ Race reaction pattern $^{a}$} & \multirow[b]{2}{*}{ Total } & \multirow[b]{2}{*}{ Gene(s) } & \multicolumn{5}{|c|}{ Number of accessions from province ${ }^{b}$} \\
\hline 1 & 3 & 4 & 5 & 7 & 10 & 12 & 17 & 20 & 25 & & & Anhui & Hubei & Jiangsu & Sichuan & Zhejiang \\
\hline $\mathrm{R}$ & $\mathrm{R}$ & $\mathrm{R}$ & $\mathrm{R}$ & $\mathrm{R}$ & $\mathrm{R}$ & $\mathrm{R}$ & $\mathrm{S}$ & $\mathrm{R}$ & $\mathrm{R}$ & Rps $1 d^{\mathrm{c}}$ & 2 & 0 & 1 & 1 & 0 & 0 \\
\hline $\mathrm{R}$ & $\mathrm{R}$ & $\mathrm{R}$ & $\mathrm{R}$ & $\mathrm{R}$ & $\mathrm{R}$ & $\mathrm{S}$ & $\mathrm{R}$ & S & $\mathrm{S}$ & Rpslk & 22 & 2 & 13 & 3 & 4 & 0 \\
\hline $\mathrm{R}$ & $\mathrm{R}$ & $\mathrm{R}$ & $\mathrm{R}$ & $\mathrm{R}$ & $\mathrm{S}$ & $\mathrm{R}$ & $\mathrm{S}$ & S & $\mathrm{R}$ & $\operatorname{Rps} 3 b^{\mathrm{c}}$ & 3 & 0 & 2 & 0 & 1 & 0 \\
\hline $\mathrm{R}$ & $\mathrm{R}$ & $\mathrm{S}$ & $\mathrm{S}$ & $\mathrm{R}$ & $\mathrm{R}$ & $\mathrm{R}$ & $\mathrm{R}$ & $\mathrm{S}$ & $\mathrm{S}$ & Rpslc & 2 & 1 & 1 & 0 & 0 & 0 \\
\hline $\mathrm{R}$ & $\mathrm{R}$ & $\mathrm{R}$ & $\mathrm{R}$ & $\mathrm{S}$ & $\mathrm{S}$ & $\mathrm{S}$ & $\mathrm{S}$ & $\mathrm{S}$ & $\mathrm{R}$ & Rps $3 a$ & 11 & 0 & 3 & 5 & 1 & 2 \\
\hline $\mathrm{R}$ & $\mathrm{S}$ & $\mathrm{S}$ & $\mathrm{S}$ & $\mathrm{S}$ & $\mathrm{R}$ & $\mathrm{S}$ & $\mathrm{R}$ & $S$ & $\mathrm{~S}$ & Rps1 & 9 & 1 & 2 & 3 & 3 & 0 \\
\hline $\mathrm{S}$ & $\mathrm{S}$ & S & $\mathrm{S}$ & $\mathrm{S}$ & $\mathrm{S}$ & $\mathrm{R}$ & $\mathrm{S}$ & $\mathrm{S}$ & $\mathrm{S}$ & Rps 7 & 16 & 0 & 2 & 8 & 6 & 0 \\
\hline $\mathrm{R}$ & $\mathrm{R}$ & $\mathrm{R}$ & $\mathrm{R}$ & $\mathrm{R}$ & $\mathrm{R}$ & $\mathrm{R}$ & $\mathrm{R}$ & $\mathrm{R}$ & $\mathrm{R}$ & $*_{\mathrm{d}}$ & 18 & 1 & 1 & 11 & 4 & 1 \\
\hline $\mathrm{R}$ & $\mathrm{R}$ & $\mathrm{R}$ & $\mathrm{R}$ & $\mathrm{R}$ & $\mathrm{R}$ & $\mathrm{R}$ & $\mathrm{R}$ & $\mathrm{S}$ & $\mathrm{R}$ & $*$ & 3 & 0 & 2 & 0 & 1 & 0 \\
\hline $\mathrm{R}$ & $\mathrm{R}$ & $\mathrm{R}$ & $\mathrm{R}$ & $\mathrm{R}$ & $\mathrm{R}$ & S & $\mathrm{R}$ & $\mathrm{R}$ & $\mathrm{R}$ & $*$ & 7 & 0 & 2 & 4 & 0 & 1 \\
\hline $\mathrm{R}$ & $\mathrm{R}$ & $\mathrm{R}$ & $\mathrm{R}$ & $\mathrm{S}$ & $\mathrm{R}$ & $\mathrm{R}$ & $\mathrm{R}$ & $\mathrm{R}$ & $\mathrm{R}$ & $\ldots$ & 3 & 0 & 1 & 1 & 1 & 0 \\
\hline $\mathrm{R}$ & $\mathrm{R}$ & $\mathrm{R}$ & $\mathrm{S}$ & $\mathrm{R}$ & $\mathrm{R}$ & $\mathrm{R}$ & $\mathrm{R}$ & $\mathrm{R}$ & $\mathrm{R}$ & $*$ & 3 & 2 & 0 & 1 & 0 & 0 \\
\hline $\mathrm{R}$ & $\mathrm{R}$ & $\mathrm{R}$ & $\mathrm{R}$ & $\mathrm{R}$ & $\mathrm{R}$ & $\mathrm{R}$ & $\mathrm{R}$ & S & $\mathrm{S}$ & $*$ & 8 & 1 & 2 & 4 & 1 & 0 \\
\hline $\mathrm{R}$ & $\mathrm{R}$ & $\mathrm{R}$ & $\mathrm{R}$ & $\mathrm{R}$ & $\mathrm{R}$ & $\mathrm{S}$ & $\mathrm{R}$ & $\mathrm{S}$ & $\mathrm{R}$ & $*$ & 24 & 1 & 13 & 4 & 4 & 2 \\
\hline $\mathrm{R}$ & $\mathrm{R}$ & $\mathrm{R}$ & $\mathrm{R}$ & $\mathrm{R}$ & $\mathrm{S}$ & $\mathrm{R}$ & $\mathrm{R}$ & $\mathrm{S}$ & $\mathrm{R}$ & $\ldots$ & 2 & 0 & 0 & 0 & 1 & 1 \\
\hline $\mathrm{R}$ & $\mathrm{R}$ & $\mathrm{R}$ & $\mathrm{R}$ & $\mathrm{S}$ & $\mathrm{R}$ & $\mathrm{R}$ & $\mathrm{S}$ & $\mathrm{R}$ & $\mathrm{R}$ & $\ldots$ & 4 & 0 & 0 & 3 & 1 & 0 \\
\hline $\mathrm{R}$ & $\mathrm{R}$ & $\mathrm{R}$ & $\mathrm{R}$ & $\mathrm{S}$ & $\mathrm{R}$ & S & $\mathrm{R}$ & $\mathrm{R}$ & $\mathrm{R}$ & $*$ & 4 & 1 & 2 & 1 & 0 & 0 \\
\hline $\mathrm{R}$ & $\mathrm{R}$ & $\mathrm{S}$ & $\mathrm{R}$ & $\mathrm{R}$ & $\mathrm{R}$ & $\mathrm{R}$ & $\mathrm{R}$ & $\mathrm{R}$ & $\mathrm{S}$ & $\ldots$ & 2 & 0 & 0 & 1 & 1 & 0 \\
\hline $\mathrm{R}$ & $\mathrm{R}$ & $\mathrm{S}$ & $\mathrm{R}$ & $\mathrm{R}$ & $\mathrm{R}$ & $\mathrm{R}$ & $\mathrm{R}$ & $\mathrm{S}$ & $\mathrm{R}$ & * & 2 & 0 & 1 & 0 & 0 & 1 \\
\hline $\mathrm{R}$ & $\mathrm{R}$ & $S$ & $\mathrm{~S}$ & $\mathrm{R}$ & $\mathrm{R}$ & $\mathrm{R}$ & $\mathrm{R}$ & $\mathrm{R}$ & $\mathrm{R}$ & $\ldots$ & 3 & 0 & 0 & 0 & 3 & 0 \\
\hline $\mathrm{R}$ & $\mathrm{R}$ & $\mathrm{R}$ & $\mathrm{R}$ & $\mathrm{R}$ & $\mathrm{R}$ & $S$ & $\mathrm{~S}$ & S & $\mathrm{R}$ & $\ldots$ & 5 & 0 & 2 & 2 & 0 & 1 \\
\hline $\mathrm{R}$ & $\mathrm{R}$ & $\mathrm{R}$ & $\mathrm{R}$ & $\mathrm{R}$ & $\mathrm{S}$ & $S$ & $\mathrm{R}$ & $S$ & $\mathrm{R}$ & $\ldots$ & 4 & 1 & 1 & 0 & 2 & 0 \\
\hline $\mathrm{R}$ & $\mathrm{R}$ & $\mathrm{R}$ & $\mathrm{R}$ & $\mathrm{S}$ & $\mathrm{S}$ & $\mathrm{R}$ & $\mathrm{S}$ & $\mathrm{R}$ & $\mathrm{R}$ & $\ldots$ & 2 & 0 & 0 & 2 & 0 & 0 \\
\hline $\mathrm{R}$ & $\mathrm{R}$ & $\mathrm{R}$ & $\mathrm{S}$ & $S$ & $\mathrm{R}$ & S & $\mathrm{R}$ & $\mathrm{R}$ & $\mathrm{R}$ & $\ldots$ & 1 & 0 & 0 & 0 & 0 & 1 \\
\hline $\mathrm{R}$ & $\mathrm{R}$ & $\mathrm{S}$ & $\mathrm{R}$ & $\mathrm{R}$ & $\mathrm{R}$ & $\mathrm{R}$ & $\mathrm{R}$ & $\mathrm{S}$ & $\mathrm{S}$ & $\ldots$ & 8 & 1 & 4 & 2 & 0 & 1 \\
\hline $\mathrm{R}$ & $\mathrm{R}$ & $\mathrm{S}$ & $\mathrm{R}$ & $\mathrm{R}$ & $\mathrm{R}$ & S & $\mathrm{R}$ & S & $\mathrm{R}$ & $\ldots$ & 4 & 1 & 3 & 0 & 0 & 0 \\
\hline $\mathrm{R}$ & $\mathrm{R}$ & $\mathrm{S}$ & $\mathrm{R}$ & $\mathrm{R}$ & $\mathrm{S}$ & $\mathrm{R}$ & $\mathrm{R}$ & $\mathrm{R}$ & $\mathrm{S}$ & $\ldots$ & 2 & 0 & 1 & 0 & 0 & 1 \\
\hline $\mathrm{R}$ & $\mathrm{S}$ & $\mathrm{R}$ & $\mathrm{R}$ & $\mathrm{S}$ & $\mathrm{R}$ & $\mathrm{R}$ & $\mathrm{S}$ & $\mathrm{R}$ & $\mathrm{R}$ & $\ldots$ & 2 & 1 & 1 & 0 & 0 & 0 \\
\hline $\mathrm{R}$ & $\mathrm{R}$ & $\mathrm{R}$ & $\mathrm{R}$ & $\mathrm{R}$ & $\mathrm{S}$ & $S$ & $\mathrm{R}$ & $\mathrm{S}$ & $\mathrm{S}$ & $\ldots$ & 3 & 1 & 1 & 0 & 0 & 1 \\
\hline $\mathrm{R}$ & $\mathrm{R}$ & $\mathrm{R}$ & $\mathrm{R}$ & $\mathrm{R}$ & $\mathrm{S}$ & $S$ & $\mathrm{~S}$ & $S$ & $\mathrm{R}$ & $*$ & 14 & 1 & 3 & 2 & 6 & 2 \\
\hline $\mathrm{R}$ & $\mathrm{R}$ & $\mathrm{R}$ & $\mathrm{R}$ & $\mathrm{S}$ & $\mathrm{R}$ & $S$ & $\mathrm{~S}$ & $S$ & $\mathrm{R}$ & $\ldots$ & 2 & 0 & 1 & 1 & 0 & 0 \\
\hline $\mathrm{R}$ & $\mathrm{R}$ & $\mathrm{R}$ & $\mathrm{R}$ & $S$ & S & $S$ & $\mathrm{R}$ & $S$ & $\mathrm{R}$ & $\ldots$ & 4 & 0 & 2 & 1 & 1 & 0 \\
\hline $\mathrm{R}$ & $\mathrm{R}$ & $\mathrm{R}$ & $\mathrm{R}$ & $\mathrm{S}$ & $\mathrm{S}$ & $S$ & $\mathrm{~S}$ & $\mathrm{R}$ & $\mathrm{R}$ & $\ldots$ & 3 & 0 & 0 & 3 & 0 & 0 \\
\hline $\mathrm{R}$ & $\mathrm{R}$ & $\mathrm{R}$ & $\mathrm{S}$ & $\mathrm{R}$ & $\mathrm{R}$ & $S$ & $\mathrm{R}$ & $\mathrm{S}$ & $\mathrm{S}$ & $\ldots$ & 2 & 0 & 2 & 0 & 0 & 0 \\
\hline $\mathrm{R}$ & $\mathrm{R}$ & S & $\mathrm{R}$ & $\mathrm{R}$ & $\mathrm{R}$ & $S$ & $\mathrm{R}$ & $S$ & $S$ & $\ldots$ & 43 & 11 & 21 & 10 & 0 & 1 \\
\hline $\mathrm{R}$ & $\mathrm{R}$ & $\mathrm{R}$ & $\mathrm{R}$ & S & $\mathrm{S}$ & S & $\mathrm{R}$ & S & $\mathrm{S}$ & $\ldots$ & 2 & 1 & 1 & 0 & 0 & 0 \\
\hline $\mathrm{R}$ & $\mathrm{R}$ & $\mathrm{R}$ & $\mathrm{R}$ & $\mathrm{R}$ & $\mathrm{S}$ & S & $\mathrm{S}$ & S & $\mathrm{S}$ & $\ldots$ & 3 & 1 & 0 & 0 & 0 & 2 \\
\hline $\mathrm{R}$ & $\mathrm{R}$ & $\mathrm{S}$ & $\mathrm{R}$ & $\mathrm{R}$ & $\mathrm{S}$ & $S$ & $\mathrm{R}$ & $S$ & $\mathrm{~S}$ & $\ldots$ & 2 & 0 & 1 & 1 & 0 & 0 \\
\hline $\mathrm{R}$ & $\mathrm{R}$ & $S$ & $\mathrm{~S}$ & $\mathrm{R}$ & $\mathrm{R}$ & $S$ & $\mathrm{R}$ & $S$ & $S$ & $\ldots$ & 15 & 6 & 4 & 2 & 2 & 1 \\
\hline $\mathrm{R}$ & $\mathrm{S}$ & $\mathrm{R}$ & $\mathrm{R}$ & $\mathrm{R}$ & $\mathrm{S}$ & S & $\mathrm{R}$ & S & $\mathrm{S}$ & $\ldots$ & 2 & 0 & 0 & 1 & 1 & 0 \\
\hline $\mathrm{R}$ & S & $\mathrm{R}$ & $\mathrm{R}$ & $\mathrm{R}$ & $\mathrm{S}$ & $S$ & $\mathrm{~S}$ & S & $\mathrm{R}$ & $\ldots$ & 2 & 0 & 0 & 2 & 0 & 0 \\
\hline $\mathrm{R}$ & $S$ & $\mathrm{R}$ & $\mathrm{R}$ & $S$ & $\mathrm{~S}$ & $\mathrm{R}$ & $\mathrm{S}$ & $S$ & $\mathrm{R}$ & $\ldots$ & 3 & 0 & 0 & 1 & 2 & 0 \\
\hline $\mathrm{R}$ & $S$ & $\mathrm{R}$ & $\mathrm{S}$ & $S$ & $S$ & $\mathrm{R}$ & $S$ & $\mathrm{R}$ & $\mathrm{R}$ & $\ldots$ & 2 & 1 & 1 & 0 & 0 & 0 \\
\hline $\mathrm{R}$ & $\mathrm{R}$ & $\mathrm{R}$ & $\mathrm{R}$ & $S$ & $\mathrm{~S}$ & S & $\mathrm{S}$ & $\mathrm{S}$ & $S$ & $\ldots$ & 7 & 0 & 5 & 2 & 0 & 0 \\
\hline $\mathrm{R}$ & $\mathrm{R}$ & $\mathrm{S}$ & $\mathrm{S}$ & $S$ & $\mathrm{R}$ & $S$ & $\mathrm{R}$ & $S$ & $\mathrm{~S}$ & $\ldots$ & 3 & 1 & 0 & 2 & 0 & 0 \\
\hline $\mathrm{R}$ & $S$ & $S$ & $\mathrm{R}$ & $S$ & $\mathrm{R}$ & $S$ & $\mathrm{R}$ & $S$ & $\mathrm{~S}$ & $\ldots$ & 6 & 1 & 0 & 3 & 2 & 0 \\
\hline $\mathrm{R}$ & $S$ & $S$ & $\mathrm{~S}$ & $S$ & $\mathrm{R}$ & $\mathrm{R}$ & $\mathrm{R}$ & $S$ & $\mathrm{~S}$ & $*$ & 2 & 0 & 0 & 2 & 0 & 0 \\
\hline $\mathrm{R}$ & $\mathrm{R}$ & $\mathrm{S}$ & $\mathrm{S}$ & $\mathrm{S}$ & $\mathrm{R}$ & S & $\mathrm{S}$ & S & $\mathrm{S}$ & $\ldots$ & 2 & 1 & 0 & 1 & 0 & 0 \\
\hline $\mathrm{R}$ & $S$ & $S$ & $\mathrm{R}$ & $S$ & $\mathrm{~S}$ & $\mathrm{R}$ & $\mathrm{S}$ & $S$ & $\mathrm{~S}$ & $\ldots$ & 2 & 0 & 1 & 1 & 0 & 0 \\
\hline $\mathrm{R}$ & $S$ & $S$ & $\mathrm{R}$ & S & $\mathrm{S}$ & S & $\mathrm{R}$ & $S$ & $\mathrm{~S}$ & $\ldots$ & 4 & 0 & 1 & 2 & 0 & 1 \\
\hline $\mathrm{R}$ & $S$ & $\mathrm{~S}$ & $\mathrm{R}$ & $\mathrm{S}$ & $\mathrm{S}$ & $S$ & $\mathrm{~S}$ & S & $\mathrm{R}$ & $\ldots$ & 2 & 0 & 0 & 0 & 0 & 2 \\
\hline S & $S$ & $\mathrm{~S}$ & $\mathrm{R}$ & $\mathrm{R}$ & $\mathrm{S}$ & $S$ & $\mathrm{R}$ & $S$ & $\mathrm{~S}$ & $\ldots$ & 3 & 0 & 0 & 0 & 3 & 0 \\
\hline $\mathrm{R}$ & $S$ & $\mathrm{R}$ & $\mathrm{S}$ & $\mathrm{S}$ & $\mathrm{S}$ & $S$ & $\mathrm{~S}$ & $S$ & S & $\ldots$ & 2 & 1 & 0 & 1 & 0 & 0 \\
\hline $\mathrm{R}$ & S & $\mathrm{S}$ & $\mathrm{R}$ & $\mathrm{S}$ & $\mathrm{S}$ & S & $S$ & S & S & $\ldots$ & 19 & 0 & 12 & 5 & 1 & 1 \\
\hline $\mathrm{R}$ & S & $S$ & $\mathrm{~S}$ & $\mathrm{~S}$ & $\mathrm{~S}$ & $\mathrm{R}$ & $\mathrm{S}$ & S & $S$ & $\ldots$ & 2 & 0 & 1 & 0 & 1 & 0 \\
\hline $\mathrm{R}$ & $S$ & $S$ & $\mathrm{~S}$ & $S$ & $\mathrm{~S}$ & $\mathrm{~S}$ & $\mathrm{R}$ & $S$ & $\mathrm{~S}$ & $\ldots$ & 2 & 0 & 0 & 0 & 2 & 0 \\
\hline $\mathrm{S}$ & $S$ & $S$ & $\mathrm{R}$ & $S$ & $\mathrm{~S}$ & $\mathrm{R}$ & $\mathrm{S}$ & $S$ & $S$ & $\ldots$ & 3 & 0 & 0 & 1 & 2 & 0 \\
\hline$S$ & $S$ & $S$ & $\mathrm{R}$ & $S$ & $\mathrm{~S}$ & S & $\mathrm{R}$ & $S$ & $S$ & $\ldots$ & 8 & 0 & 2 & 1 & 3 & 2 \\
\hline$S$ & $S$ & $\mathrm{~S}$ & $\mathrm{R}$ & $S$ & $\mathrm{~S}$ & $S$ & $\mathrm{~S}$ & $S$ & $\mathrm{R}$ & $\ldots$ & 2 & 0 & 0 & 0 & 1 & 1 \\
\hline$S$ & $S$ & $S$ & $\mathrm{~S}$ & $\mathrm{R}$ & $\mathrm{S}$ & $S$ & $\mathrm{R}$ & $S$ & $\mathrm{~S}$ & $\ldots$ & 2 & 0 & 0 & 0 & 2 & 0 \\
\hline$S$ & $S$ & $S$ & $S$ & $\mathrm{~S}$ & $S$ & $S$ & $\mathrm{R}$ & $S$ & $\mathrm{R}$ & $\ldots$ & 2 & 0 & 0 & 1 & 1 & 0 \\
\hline $\mathrm{R}$ & S & $\mathrm{S}$ & $\mathrm{S}$ & $\mathrm{S}$ & $\mathrm{S}$ & S & $\mathrm{S}$ & S & $S$ & $\ldots$ & 18 & 1 & 7 & 5 & 2 & 3 \\
\hline S & $\mathrm{R}$ & $S$ & $\mathrm{~S}$ & $S$ & $\mathrm{~S}$ & $S$ & $\mathrm{~S}$ & $S$ & $\mathrm{~S}$ & $\ldots$ & 2 & 0 & 1 & 0 & 0 & 1 \\
\hline$S$ & $S$ & $\mathrm{R}$ & $S$ & $S$ & $S$ & $S$ & $S$ & $S$ & $S$ & $\ldots$ & 2 & 0 & 1 & 0 & 1 & 0 \\
\hline$S$ & S & $\mathrm{S}$ & $\mathrm{R}$ & S & $\mathrm{S}$ & $S$ & $\mathrm{~S}$ & $S$ & $\mathrm{~S}$ & $\ldots$ & 12 & 2 & 2 & 4 & 1 & 3 \\
\hline$S$ & S & $\mathrm{S}$ & $\mathrm{S}$ & $\mathrm{S}$ & $\mathrm{S}$ & S & $\mathrm{R}$ & S & $\mathrm{S}$ & $\ldots$ & 25 & 2 & 6 & 4 & 11 & 2 \\
\hline S & $S$ & $S$ & $\mathrm{~S}$ & S & $\mathrm{S}$ & $S$ & $\mathrm{~S}$ & $\mathrm{~S}$ & $\mathrm{R}$ & $\ldots$ & 9 & 1 & 2 & 1 & 5 & 0 \\
\hline S & $\mathrm{S}$ & $\mathrm{S}$ & $\mathrm{S}$ & $\mathrm{S}$ & $\mathrm{S}$ & S & $\mathrm{S}$ & $\mathrm{S}$ & $\mathrm{S}$ & $\ldots$ & 114 & 12 & 17 & 33 & 28 & 24 \\
\hline \multicolumn{10}{|c|}{ Other reaction types } & & 79 & 11 & 18 & 18 & 18 & 14 \\
\hline & cces & & & & & & & & & & 612 & 69 & 173 & 167 & 130 & 73 \\
\hline
\end{tabular}

${ }^{\mathrm{a}} \mathrm{R}=$ resistant and intermediate, $\mathrm{S}=$ susceptible.

${ }^{\mathrm{b}}$ Data for the nine accessions from Guangdong and the seven accessions from Shanghai has not been included. These accessions were susceptible all or most races used for evaluation.

${ }^{\mathrm{c}}$ Reaction type may be achieved with a single resistance gene or a combination of two previously described resistance genes.

$\mathrm{d} *$, Reaction type may be achieved with a combination of two previously described resistance genes. 
on resistant $(\mathrm{R})$ or susceptible $(\mathrm{S})$ reactions to each of the 10 races of $P$. sojae. It is believed factors like CNS-type reactions (17) of resistance, poor seed quality, and temperature increased the number of susceptible plants of some accessions, causing intermediate classifications (21). The CNS-type response is identified by using hydroponic inoculation (17), and with the hypocotyl inoculation technique, responses of the plant can vary. Intermediate reactions were combined with resistant reactions for classification of reaction types based on this reasoning. Reaction types that occurred more than once are listed in Table 3. The number of accessions for each reaction type is given in total and by province. Reaction types have been identified which are consistent with identified single genes and gene combinations based on the response of the differential cultivars to the cultures of $P$. sojae used.

Reaction types of accessions that are consistent with the reaction types of a single gene are listed at the top of Table 3 . The reaction type $R_{1} R_{3} R_{4} R_{5} R_{7} R_{10} R_{12} S_{17}$ $R_{20} R_{25}$ is consistent with the reaction type of Rpsld (10) and several two-gene combinations. The $\mathrm{R}_{1} \mathrm{R}_{3} \mathrm{R}_{4} \mathrm{R}_{5} \mathrm{R}_{7} \mathrm{R}_{10} \mathrm{~S}_{12} \mathrm{R}_{17}$ $\mathrm{S}_{20} \mathrm{~S}_{25}$ reaction type, which is consistent with Rpslk (6) reaction, occurred in 22 accessions. The number of provinces donating these accessions suggests that Rpslk-type resistance exists in several areas of China. Reaction types were also identified that were consistent with the Rps3b (25), Rpslc (22), Rps3a (4,22), Rps1 (8), and Rps7 (2) genes for resistance.

The $R_{1} R_{3} R_{4} R_{5} R_{7} R_{10} R_{12} R_{17} R_{20} R_{25}$ reaction type occurred in 18 accessions. Eleven of these accessions were from the province of Jiangsu and four were from Sichuan. This reaction type is not conferred by any single previously identified gene, but is consistent with the gene combinations of Rps1k with Rps4 (5) or Rps6 (3). The $R_{1} R_{3} R_{4} R_{5} R_{7} R_{10} R_{12} R_{17} S_{20} R_{25}$ reaction type, which is consistent with several gene combinations, occurred in only three accessions; two from Hubei and one from Sichuan. The $R_{1} R_{3} R_{4} R_{5} R_{7} R_{10} S_{12} R_{17} R_{20} R_{25}$ reaction type occurred in seven accessions; four from Jiangsu, two from Hubei, and one from Zhejiang. This reaction type offers resistance to the more recently identified races 17,20 , and 25 , and is consistent with the reaction type of Rpslk in combi- nation with Rps2 (17). The reaction type, $R_{1} R_{3} R_{4} R_{5} S_{7} R_{10} R_{12} R_{17} R_{20} R_{25}$ occurred in one accession from each of the provinces of Hubei, Jiangsu, and Sichuan. This reaction type provides resistance to the highly virulent races $12,17,20$, and 25 , and is not consistent with any identified gene or twogene combinations. The $\mathrm{R}_{1} \mathrm{R}_{3} \mathrm{R}_{4} \mathrm{~S}_{5} \mathrm{R}_{7} \mathrm{R}_{10} \mathrm{R}_{12}$ $R_{17} R_{20} R_{25}$ reaction type occurred in two accessions from Anhui and one from $\mathrm{Ji}$ angsu. This reaction type is consistent with the three possible combinations of the Rps1c gene with Rps2, Rps4, or Rps6. These accessions also provide resistance to the highly virulent races capable of overcoming commonly used resistance genes.

Other resistant reaction types are not discussed individually. Forty-nine accessions were resistant to 8 of the 10 races. Of these, 24 accessions gave a reaction type that is consistent with the two combinations of the Rpslk gene with Rps $3 a$ or Rps5 (9). Fifty-three accessions had reaction types with resistance to 7 of the 10 races, including those with $R p s l k$ reaction types. The largest proportion of these accessions were from the provinces of Hubei. Resistance to 6 of the 10 races occurred in 73 accessions, with the majority from the provinces of Anhui, Hubei, and Jiangsu. Forty-two accessions were resistant to half of the races, including 11 accessions with a reaction type consistent with Rps3a. Eighteen accessions, distributed between four reaction types, were resistant to 4 of the 10 races. Resistance to 3 of the 10 races occurred in 22 accessions, including nine accessions with a reaction type consistent with Rps1. Forty-two accessions were resistant to 2 of the 10 races. Susceptibility to all but 1 of the 10 races occurred in 84 accessions. Seventeen of these accessions conferred resistant reactions to only race 12 , which is consistent with the Rps7 reaction type. The most common reaction type, $\mathrm{S}_{1} \mathrm{~S}_{3} \mathrm{~S}_{4} \mathrm{~S}_{5} \mathrm{~S}_{7} \mathrm{~S}_{10} \mathrm{~S}_{12} \mathrm{~S}_{17} \mathrm{~S}_{20} \mathrm{~S}_{25}$, was conferred by 114 accessions. The province of Hubei had 17 accessions, or $10 \%$, with the $\mathrm{S}_{1} \mathrm{~S}_{3} \mathrm{~S}_{4} \mathrm{~S}_{5} \mathrm{~S}_{7} \mathrm{~S}_{10} \mathrm{~S}_{12} \mathrm{~S}_{17} \mathrm{~S}_{20} \mathrm{~S}_{25}$ reaction type. It is probable that Phytophthora resistance is crucial to soybean in provinces where a low percentage of the accessions had this reaction type.

From the numerous reaction types that occurred in soybean accessions, it is apparent that Phytophthora resistance is extremely diverse in China. Accessions with resistance to 8 or more of the 10 races occurred most frequently in accessions from the provinces of Hubei, Jiangsu, and Sichuan. The provinces of Hubei and Jiangsu are located in eastern China, where annual precipitation may reach $2,000 \mathrm{~mm}$ (20). In Hubei, $2.2 \%$ of the cultivated land is used for soybean production. In Jiangsu, $12.1 \%$ of the cultivated land is used for soybean production (13). The wet conditions and soybean production in the provinces of Hubei and Jiangsu have likely affected the level and incidence of Phytophthora resistance in accessions from these provinces. The province of Sichuan is located in central China. The majority of Sichuan receives 1,000 to $2,000 \mathrm{~mm}$ precipitation annually, with areas receiving less than $250 \mathrm{~mm}$ and some receiving greater than $2,500 \mathrm{~mm}$ (20). It is probable that the accessions from Sichuan with Phytophthora resistance were collected from areas of high annual precipitation, where disease pressure is more likely to occur. Further collection efforts for Phytophthora-resistant soybean should be focused in the provinces of Hubei, Jiangsu, and Sichuan.

Accessions with resistance to at least 9 of the 10 races used for evaluation have been listed in Table 4. Accessions with $R_{1} R_{3} R_{4} R_{5} R_{7} R_{10} R_{12} R_{17} R_{20} R_{25}$ reaction types should receive precedence for further evaluation and implementation of these sources into breeding programs. Of the 18 accessions that conferred this reaction type, six accessions have been selected based on the suitability of their plant characteristics. Accessions PI 587.550A to 588.053B from southern China are maturity group $\mathrm{V}$ or later. PI 587.612B, 587.612C, 588.006C, 588.017B, 588.017C, and 588.023A were selected for breeding programs based on $R_{1} R_{3} R_{4} R_{5} R_{7} R_{10} R_{12} R_{17}$ $R_{20} R_{25}$ reaction types, erect or semiappressed pubescence, normal pubescence density, and yellow seed coat color.

Accessions with high levels of resistance should be evaluated further to determine the nature of their resistance. New resistance genes identified in these accessions from China may enable soybean producers to have resistance as a usable management strategy in the future.

\section{LITERATURE CITED}

1. Abney, T, S., Melgar, J. C., Richards, T. L., Scott, D. H., Grogan, J. and Young, J. 1997.

Table 4. Accessions with resistant responses to 9 or more of the 10 races of Phytophthora sojae used for evaluation

\begin{tabular}{ll}
\hline Reaction type & \multicolumn{1}{c}{ Accessions } \\
\hline $\mathrm{R}_{1} \mathrm{R}_{3} \mathrm{R}_{4} \mathrm{R}_{5} \mathrm{R}_{7} \mathrm{R}_{10} \mathrm{R}_{12} \mathrm{R}_{17} \mathrm{R}_{20} \mathrm{R}_{25}$ & PI 587.557B, PI 587.580B, PI 587.612C, PI 588.006A, PI 587.565A, PI 587.600C, PI 587.704, PI 588.017B, \\
& $\begin{array}{l}\text { PI 587.565C, PI 587.604D, PI 587.837, PI 588.017C, PI 587.567C, PI 587.612A, PI 587.852, PI 588.023A, PI 587.571, } \\
\end{array}$ \\
& PI 587.612B \\
$\mathrm{R}_{1} \mathrm{R}_{3} \mathrm{R}_{4} \mathrm{R}_{5} \mathrm{R}_{7} \mathrm{R}_{10} \mathrm{R}_{12} \mathrm{R}_{17} \mathrm{~S}_{20} \mathrm{R}_{25}$ & PI 587.832, PI 587.844A, PI 588.016 \\
$\mathrm{R}_{1} \mathrm{R}_{3} \mathrm{R}_{4} \mathrm{R}_{5} \mathrm{R}_{7} \mathrm{R}_{10} \mathrm{R}_{12} \mathrm{~S}_{17} \mathrm{R}_{20} \mathrm{R}_{25}$ & PI 587.550A, PI 587.714A \\
$\mathrm{R}_{1} \mathrm{R}_{3} \mathrm{R}_{4} \mathrm{R}_{5} \mathrm{R}_{7} \mathrm{R}_{10} \mathrm{~S}_{12} \mathrm{R}_{17} \mathrm{R}_{20} \mathrm{R}_{25}$ & PI 587.567B, PI 587.572B, PI 587.719B, PI 587.889, PI 587.570A, PI 587.595C, PI 587.815A \\
$\mathrm{R}_{1} \mathrm{R}_{3} \mathrm{R}_{4} \mathrm{R}_{5} \mathrm{~S}_{7} \mathrm{R}_{10} \mathrm{R}_{12} \mathrm{R}_{17} \mathrm{R}_{20} \mathrm{R}_{25}$ & PI 587.633B, PI 587.775, PI 588.006B \\
$\mathrm{R}_{1} \mathrm{R}_{3} \mathrm{R}_{4} \mathrm{~S}_{5} \mathrm{R}_{7} \mathrm{R}_{10} \mathrm{R}_{12} \mathrm{R}_{17} \mathrm{R}_{20} \mathrm{R}_{25}$ & PI 587.550B, PI 587.659A, PI 587.697 \\
\hline
\end{tabular}


New races of Phytophthora sojae with Rps1-d virulence. Plant Dis. 81:653-655.

2. Anderson, T. R., and Buzzell, R. I. 1992. Inheritance and linkage of the Rps 7 gene for resistance to Phytophthora rot of soybean. Plant Dis. 76:958-959.

3. Athow, K. L., and Laviolette, F. A. 1982. Rps6, a major gene for resistance to Phytophthora megasperma f. sp. glycinea. Phytopathology 72:1564-1567.

4. Athow, K. L., Laviolette, F. A., and Wilcox, J. R. 1979. Genetics of resistance to physiologic races of Phytophthora megasperma var. sojae in soybean cultivar, "Tracy." Phytopathology 69:641-642.

5. Athow, K. L., Laviolette, F. A., and Wilcox J. R. 1980. A new major gene for resistance to Phytophthora megasperma var. sojae in soybean. Phytopathology 70:977-980.

6. Bernard, R. L., and Cremeens, C. R. 1981. An allele at the rps1 locus from the variety "Kingwa." Soybean Genet. News1. 8:40-42.

7. Bernard, R. L., and Lindahl, D. A. 1972. Registration of "Williams" soybean. Crop Sci. 12:716.

8. Bernard, R. L., Smith, P. E., Kaufmann, M. J., and Schmitthenner, A. F. 1957. Inheritance of resistance to Phytophthora root and stem rot in soybean. Agron. J. 49:391.

9. Buzzell, R. I., and Anderson, T. R. 1981. Another major gene for resistance to Phytophthora megasperma var. sojae in soybean. Soybean Genet. Newsl. 8:30-33.

10. Buzzell, R. I., and Anderson, T. R. 1992. Inheritance and race reaction of a new soybean Rps1 allele. Plant Dis. 76:600-601.

11. Buzzell, R. I., Anderson, T.R., and Rennie, B. D. 1987. Harosoy Rps isolines. Soybean Genet. Newsl. 14:79-81.

12. Doupnik, B., Jr. 1993. Soybean production and disease loss estimates for the North Central United States from 1989-1991. Plant Dis. 77:1170-1171.

13. Editorial Board of China Agricultural Yearbook. 1987. China Agricultural Yearbook 1986, First Edition. Agricultural Publishing House, Beijing, China.
14. Faris, M. A., and Sabo, F. E. 1989. The systematics of Phytophthora sojae and P. megasperma. Can. J. of Bot. 67:1442.

15. Henry, R. N., and Kirkpatrick, T. L. 1995. Two new races of Phytophthora sojae, causal agent of Phytophthora root and stem rot of soybean, identified from Arkansas soybean fields. Plant Dis. 79:1074.

16. Kaufmann, M. J., and Gerdemann, J. W. 1958. Root and stem rot of soybean caused by Phytophthora sojae N. SP. Phytopathology 48:201-208.

17. Kilen, T. C., Hartwig, E. E., and Keeling, B. L. 1974. Inheritance of a second major gene for resistance to Phytophthora rot in soybeans. Crop Sci. 14:260-262.

18. Lohnes, D. G., Nickell, C. D., and Schmitthenner, A. F. 1996. Origin of soybean alleles for Phytophthora resistance in China. Crop Sci. 36:1689-1692.

19. McBlain, B. A., Fioritto, R. J., St. Martin, S. K., Galip-DuBois, A., Schmitthenner, A. F., Cooper, R. L., and Martin, R. J. 1991. Registration of "Chapman" soybean. Crop Sci. 31:487-488.

20. Medici, G. (ed.) 1973. World Atlas of Agriculture-Vol. 2. Asia and Oceania. Istituto Geografico De Agostini, Novara, Italy.

21. Moots, C. K., Nickell, C. D., Gray, L. E., and Lim, S. M. 1983. Reaction of soybean cultivars to 14 races of Phytophthora megasperma f. sp. glycinea. Plant Dis. 67:764-767.

22. Mueller, E. H., Athow, K. L., and Laviolette, F. A. 1978. Inheritance of resistance to four physiological races of Phytophthora megasperma var. sojae. Phytopathology 68:13181322.

23. Nickell, C. D., Thomas, D. J., and Frey, K. 1994. Registration of "Piatt" soybean. Crop Sci. 34:1128-1129.

24. Nickell, C. D., Thomas, D. J., Cary, T. R., and Heavner, D. 1996. Registration of "Macon" soybean. Crop Sci. 36:1410.

25. Ploper, L. D., Athow, K. L., and Laviolette, F. A. 1985. A new allele at the Rps 3 locus for resistance to Phytophthora megasperma f. sp. glycinea in soybean. Phytopathology 75:690-
694.

26. Rennie, B. D., Buzzell, R. I., Anderson, T. R., and Beversdorf, W. D. 1992. Evaluation of four Japanese soybean cultivars for Rps alleles conferring resistance to Phytophthora megasperma f. sp. glycinea. Can. J. Plant Sci. 72:217-220.

27. Rutherford, F. S., Ward, E. W. B., and Buzzell, R. I. 1985. Variation in virulence in successive single zoospore propagations of Phytophthora megasperma f. sp. glycinea. Phytopathology 75:371-374.

28. Schmitthenner, A. F. 1985. Problems and progress in control of Phytophthora root rot of soybean. Plant Dis. 69:362-368.

29. Schmitthenner, A. F., and Bhat, R. G. 1994 Useful methods for studying Phytophthora in the laboratory. Dep. Plant Pathol. Ohio Agric. Res. Dev. Cent. Circ. 143

30. Schmitthenner, A. F., Hobe, M., and Bhat, R. G. 1994. Phytophthora sojae races in Ohio over a ten year interval. Plant Dis. 78:269 276.

31. Wagner, R. E., and Bernard, R. L. 1991. Response of "Williams" isolines to eight races of Phytophthora megasperma f. sp. glycinea. Soybean Genet. Newsl. 18:236-239.

32. Ward, E. W. B. 1990. The interaction of soya beans with Phytophthora megasperma f. sp. glycinea. p 299-310. In: Biological Control of Soil-Borne Plant Pathogens. D. Hornby, ed. C. A. B. International, Wallingford, England.

33. Weiss, M. G. and Stevenson, T. M. 1955 Registration of soybean varieties, V. Agron. J. 47:541-543.

34. Wrather, J. A., Anderson, T. R., Arsyad, D. M., Gai, J., Ploper, L. D., Porta-Puglia, A., Ram, H. H., and Yorinri, J. T. 1997. Soybean disease loss estimates for the top 10 soybean producing countries in 1994. Plant Dis 81:107-110.

35. Yanchun, S., and Chongyao, S. 1993. The discovery and biological characteristics studies of Phytophthora megasperma f. sp. gly cinea on soybean in China. Acta Phytopathol. Sin. 23:341-347. 